\title{
Soret-Dufour Effects on Hydromagnetic Non-Darcy Convective-Radiative Heat and Mass Transfer over a Stretching Sheet in Porous Medium with Viscous Dissipation and Ohmic Heating
}

\author{
D. $\mathrm{Pal}^{1 \dagger}$ and $\mathrm{H}$. Mondal ${ }^{2}$ \\ ${ }^{I}$ Department of Mathematics, Siksha-Bhavana, Visva-Bharati University, Santiniketan, West Bengal-731235, \\ India \\ ${ }^{2}$ Department of Mathematics, Bengal Institute of Technology and Management, West Bengal-731236, India
}

†Corresponding Author Email: dulalp123@ rediffmail.com

(Received May 11, 2013; accepted June 10, 2013)

\begin{abstract}
The present study is devoted to investigate the effects of Soret and Dufour on the mixed convection flow, heat and mass transfer over a stretching sheet in the presence of viscous dissipation, Ohmic heating, thermal radiation in porous medium. Numerical solutions for the coupled governing equations are obtained by using the fifth-order Runge-Kutta-Fehlberg method with shooting technique. Important features of flow, heat and mass transfer characteristics for different values of the physical parameters are analyzed and discussed. Numerical results reveal that the magnetic field and inertia coefficient reduce the skin friction but reverse effects are seen on local Nusselt number.
\end{abstract}

Keywords: Magnetohydrodynamics, Thermal radiation, Stretching sheet, Dufour and Soret effect, Porous medium,Convection.

\section{NOMENCLATURE}

$\begin{array}{ll}A_{O} & \text { stretching parameters on temperature } \\ A_{l} & \text { stretching parameters on concentration } \\ \mathrm{b} & \text { stretching parameter } \\ \vec{B} & \text { transverse magnetic field } \\ B_{0} & \text { uniform transverse magnetic field } \\ \mathrm{C} & \text { concentration of the species } \\ \mathrm{C}_{\mathrm{f}} & \text { local skin-friction coefficient } \\ \mathrm{Cp} & \text { specific heat at constant pressure } \\ \mathrm{D}_{\mathrm{m}} & \text { mass diffusion coefficient } \\ \mathrm{D}_{\mathrm{f}} & \text { Dufour number } \\ \vec{E} & \text { electric field } \\ \mathrm{Ec} & \text { Eckert number } \\ \mathrm{E}_{0} & \text { uniform electric field } \\ \mathrm{E}_{1} & \text { local electromagnetic parameter } \\ \mathrm{F} & \text { empirical constant } \\ \mathrm{F}^{*} & \text { local inertia-coefficient } \\ G r_{x} & \text { Grashof number } \\ \mathrm{g} & \text { acceleration due to gravity } \\ \mathrm{Ha} & \text { Hartmann number } \\ \mathrm{k} & \text { permeability of the porous medium } \\ \mathrm{k}_{1} & \text { porous parameter } \\ \mathrm{K} & \text { mean absorption coefficient } \\ \mathrm{Nr} & \text { thermal radiation parameter } \\ \mathrm{Pr} & \text { Prandtl number }\end{array}$

$\mathrm{q}_{\mathrm{r}} \quad$ radiative heat flux

$\operatorname{Re}_{x}$ local Reynolds number

Sc Schmidt number

$S h_{x}$ local Sherwood number

$\mathrm{Sr} \quad$ Soret number

$\mathrm{T}$ temperature of the fluid

$\mathrm{T}_{\mathrm{w}} \quad$ stretching sheet temperature

$T_{\infty} \quad$ temperature far away from stretching sheet

$\mathrm{Tm}$ mean fluid tempetature

$\mathrm{u}$ velocity of the fluid in the $\mathrm{x}$-direction

$\mathrm{v}$ velocity of the fluid in the $y$-direction

$\mathrm{x}$ flow directional coordinate along the the stretching sheet

y distance normal to the stretching sheet

$\theta$ non-dimensional temperature parameter

$\beta \quad$ co-efficient of thermal expansion

$\eta \quad$ similarity variable

$v \quad$ kinematic viscosity

$\rho$ density of the fluid

$\kappa \quad$ thermal conductivity

$\delta \quad$ solutal buoyany parameter

$\lambda$ buoyancy parameter 


\section{INTRODUCTION}

Convective heat and mass transfer phenomena in fluid saturated porous media over stretching surface has been of growing interest during the last several decades because of its great practical applications in many areas, such as continuous casting, drawing of plastic sheets, metal and polymer extrusion etc. Crane (1970) studied the steady two-dimensional boundary layer flow due to the stretching of a flat elastic sheet. Minto et al. (1998) have considered free convection flow on a vertical surface embedded in the porous medium. Ali (2007) analyzed the effect of lateral mass flux on the natural convection boundary layer induced by a heated vertical plate embedded in a saturated porous medium with an exponential decaying heat generation.

All the above mentioned investigations are confined to Darcy's law which states that the volumeaveraged velocity is proportional to the pressure gradient. The Darcy model is valid in a densely packed porous medium made up of uniform spherical particles under condition of low velocity and small porosity and permeability. However, for relatively high velocity flow situations, the Darcy law is inadequate to represent the flow behavior correctly since it does not account inertia effects of the porous medium. In such case, the pressure drop has a quadratic relationship with volumetric flow rate. Several investigators have considered the nonDarcian model to study convective heat transfer in a porous medium for Newtonian fluid. Vafai and Tien (1981) discussed the importance of inertia effects for high velocity flow in porous media. Bejan and Poulikakos (1984) used Forchheimer,s model to study natural convection in a porous medium. The boundary effects can be included by adding viscous shear term and adding a non-linear term in the momentum equation to account for drag effects as noted by Nield and Bejan (2006). Rudraiah (1984) investigated convection in a porous medium by incorporating the boundary and inertia effects. Applying the boundary layer approach, some authors have reported analytical solution for the Brinkman Forchheimer momentum equation by Vafai and Kim (1989).

A new dimension is added to the study of flow and heat transfer in a viscous fluid over a stretching surface in the presence of thermal radiation. The radiative effects have important applications in physics and engineering particularly in space technology and high temperature processes. Thermal radiation effect might play a significant role in controlling heat transfer process in polymer processing industry. Bakier and Gorla (1996) investigated the effect of thermal radiation on mixed convection from horizontal surfaces in saturated porous media.The quality of the final product depends to a great extent on the heat controlling factors and the knowledge of radiative heat transfer in the system can perhaps lead to a desired product with a sought characteristic. Pal and Malashetty (2008) have presented similarity solutions of the boundary layer equations to analyze the effects of thermal radiation on stagnation point flow over a stretching sheet with internal heat generation or absorption. Pal (2009) investigated the effect of thermal radiation on heat and mass transfer in two-dimensional stagnation point flow of an incompressible viscous fluid over a stretching sheet in the presence of buoyancy force. Recently, Pal and Mondal (2011) analyzed the influence of thermal radiation on hydromagnetic Darcy-Forchheimer mixed convection flow past a stretching sheet embedded in a porous medium. Recently, Anjalidevi and Kayalvizhi (2013) investigated nonlinear hydromagnetic flow with radiation and heat source over a stretching surface with prescribed heat and mass flux embedded in a porous medium.

Some specific industrial applications such as in polymer processing technology that involves cooling of continuous strip or filaments. During the process, strips are sometimes stretched. The properties of the final product depend on the rate of cooling. The rate of cooling can be controlled by the use of electrically conducting fluid with the application of the magnetic field. Numerous attempts have been made to analyze the effects of transverse magnetic field on the boundary layer flow, heat and mass transfer characteristics of electrically conducting fluid. Vajravelu and Rollins (1992) studied heat transfer in an electrically conducting fluid over a stretching surface by taking into account of magnetic field. Mostafa et al. (2012) investigated the MHD flow and heat transfer of a micropolar fluid over a stretching surface with heat generation/absorption and slip velocity.

It is well known that the heat transfer due to concentration gradient is called the Dufour effect (or diffusion-thermo) whereas the mass transfer caused by temperature gradient is called Soret effect (or thermal-diffusion). In other words, Soret effect is referred to the species differentiation developed in an initial homogeneous mixture submitted to a thermal gradient whereas Dufour effect is referred to heat flux produced by the concentration gradient. Alam et al. (2006) studied the Soret and Dufour effects on a steady MHD combined free-forced convective and mass transfer flow past a semiinfinite vertical plate. Postelnicu (2004) discussed the influence of a magnetic field on heat and mass transfer by natural convection from a vertical surface in porous media in the presence of Soret and Dufour effects. Pal and Chatterjee (2011) analyzed mixed convection magnetohydrodynamic heat and mass transfer past a stretching surface in a micropolar fluid--saturated porous medium in the presence of Ohmic heating, Soret and Dufour effects. Reddy and Rao (2012) analyzed thermodiffusion and diffusion-thermo effects on convective heat and mass transfer through a porous medium in a circular cylindrical annulus with quadratic density temperature variation. Recently, Gangadha (2013) studied Soret and Dufour effects on hydro magnetic heat and mass transfer over a vertical plate with a convective surface boundary condition and chemical reaction.

In all these works effect of electric field has been neglected which is one of the important parameters to alter the momentum and heat transfer 
characteristics in a Newtonian boundary layer flow. Thus in view of the above investigations, authors envisage to analyzed the effects of thermal radiation, viscous dissipation and Ohmic heating on MHD non-Darcy mass diffusion of species over a continuous stretching sheet in the presence of variable electric and magnetic fields with Soret and Dufour effects. Highly non-linear momentum, energy and mass-diffusion equations are solved numerically using fifth-order Runge-KuttaFehlberg method with shooting technique by $\mathrm{Na}$ (1979).

\section{MATHEMATICAL FORMULATIONS}

We consider two-dimensional steady incompressible electrically conducting fluid flow over a continuous stretching sheet embedded in a porous medium in the presence of mass diffusion of species. The flow region is exposed under uniform transverse magnetic field $\vec{B}=\left(0, B_{0}, 0\right)$ and variable electric field $\vec{E}=\left(0,0,-E_{0}(x)\right)$ since such imposition of variable electric and magnetic fields stabilizes the boundary layer flow. It is assumed that the flow is generated by stretching of an elastic boundary sheet from a slot by imposing two equal and opposite forces in such a way that velocity of the boundary sheet is of linear order of the flow direction (see Fig.1).

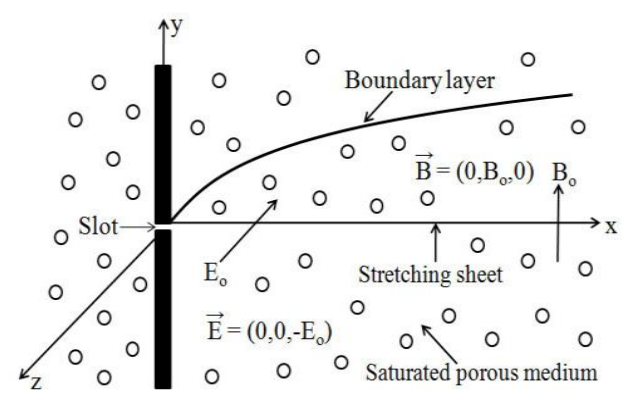

Fig.1. Boundary layer over stretching sheet

We know from Maxwell's equation that $\nabla \cdot \vec{B}=0$ and $\nabla \times \vec{E}=0$. When magnetic field is not so strong then electric field and magnetic field obey Ohm's law $\vec{J}=\sigma(\vec{E}+\vec{q} \times \vec{B})$ where $\vec{J}$ is the Joule current, $\sigma$ is the magnetic permeability and $\vec{q}$ is the fluid velocity. We assume that magnetic Reynolds number of the fluid is small so that induced magnetic field and Hall effect are neglected. The governing boundary layer equations under Boussinesq's approximation are

(i) Conservation of mass

$\frac{\partial u}{\partial x}+\frac{\partial v}{\partial y}=0$ (ii) Conservation of momentum

$$
\begin{aligned}
& u \frac{\partial u}{\partial x}+v \frac{\partial u}{\partial y}=v \frac{\partial^{2} u}{\partial y^{2}}+\frac{\sigma}{\rho}\left(E_{0}(x) B_{0}-B_{0}{ }^{2} u\right) \\
& -\frac{v}{k} u-\frac{C_{b}}{\sqrt{k}} u^{2}+g \beta_{T}\left(T-T_{\infty}\right)+g \beta_{c}\left(C-C_{\infty}\right)
\end{aligned}
$$

(iii) Energy Equation

$$
\begin{aligned}
& u \frac{\partial T}{\partial x}+v \frac{\partial T}{\partial y}=\frac{\kappa}{\rho C_{p}} \frac{\partial^{2} T}{\partial y^{2}} \\
& +\frac{\mu}{\rho C_{p}}\left(\frac{\partial u}{\partial y}\right)^{2}+\frac{\sigma}{\rho C_{p}}\left(u B_{0}-E_{0}(x)\right)^{2} \\
& -\frac{1}{\rho C_{p}} \frac{\partial q_{r}}{\partial y}+\frac{D_{m} K_{T}}{C_{s} C_{p}} \frac{\partial^{2} C}{\partial y^{2}}
\end{aligned}
$$

(iv) Conservation of species

$u \frac{\partial C}{\partial x}+v \frac{\partial C}{\partial y}=D_{m} \frac{\partial^{2} C}{\partial y^{2}}+\frac{D_{m} K_{T}}{T_{m}} \frac{\partial^{2} T}{\partial y^{2}}$

The boundary conditions for Eqs.. (1) to(4) are expressed as

$$
\begin{aligned}
& u=U_{w}(x)=b x, v=0, \\
& T=T_{w}=T_{\infty}+A_{0}\left(\frac{x}{l}\right)^{2}, C=C_{w} \\
& =C_{\infty}+A_{1}\left(\frac{x}{l}\right)^{2} \quad \text { at } y=0 \\
& u=0, T \rightarrow T_{\infty}, C \rightarrow C_{\infty} \text { as } y \rightarrow \infty
\end{aligned}
$$

where $\mathrm{u}$ and $\mathrm{v}$ are the velocity components in the $\mathrm{x}$ and y-directions respectively; $v$ is the kinematic viscosity, $C_{\infty}$ is the concentration of species in the free stream, $T_{w}$ stands for stretching sheet temperature, $T_{\infty}$ is the temperature far away from the stretching sheet, $C_{w}$ stands for concentration at the wall and $E_{0}(x)=E x$ is a variable electric field and $\mathrm{E}$ is the constant electric parameter. In Eq. (2), fourth term on the r.h.s is added to include the inertial effects due to Forchheimer. Fourth term on the r.h.s. of Eq. (3) is related to the thermal radiation term whereas the fifth term is the Dufour term. In Eq. (5), second term on the r.h.s. indicates Soret effects. To solve the governing boundary layer Eq. (2) to (4), the following similarity transformations are introduced as

$u=b x f^{\prime}(\eta), v=-\sqrt{\frac{b}{v}} y$
$\theta=\frac{T-T_{\infty}}{T_{w}-T_{\infty}}, \phi=\frac{C-C_{\infty}}{C_{w}-C_{\infty}}$.

The thermal radiation heat flux $q_{r}$ is employed according to Rosseland approximation such that

$q_{r}=\frac{4 \sigma^{*}}{3 K} \frac{\partial T^{4}}{\partial y}$ 
where $\sigma^{*}$ and $\mathrm{K}$ are the Stefan-Boltzmann constant and the mean absorption coefficient, respectively. The fluid-phase temperature differences within the flow are assumed to be sufficient small so that $T^{4}$ may be expressed as a linear function of temperature. This is done by expanding $T^{4}$ in a Taylor series about the free stream temperature $T_{\infty}$ and neglecting higher order terms to yield

$T^{4}=4 T_{\infty}^{3} T-3 T_{\infty}^{4}$.

Substitution of Eqs. (6) and (7) into the governing Eqs. (2) to (4) and using the above relations we finally obtain a system of non-linear ordinary differential equations with appropriate boundary conditions

$$
\begin{aligned}
& f^{\prime 2}-f f^{\prime \prime}=f^{\prime \prime \prime}+H a^{2}\left(E_{1}-f^{\prime}\right) \\
& -k_{1} f^{\prime}-F^{*} f^{\prime 2}+\lambda \theta+\delta \phi \\
& \frac{1+N r}{\operatorname{Pr}} \theta^{\prime \prime}+\left(f \theta^{\prime}-2 f^{\prime} \theta\right)= \\
& -E c f^{\prime \prime 2}-E c H a^{2}\left(f^{\prime}-E_{1}\right)^{2}-D_{f} \phi^{\prime \prime} \\
& \phi^{\prime \prime}+S c\left(f \phi^{\prime}-2 f^{\prime} \phi\right)=-S c S r \theta^{\prime \prime} .
\end{aligned}
$$

The boundary conditions (9)-(11) becomes $f(0)=0, f^{\prime}(0)=1, \theta(0)=1, \phi(0)=1$ at $\eta=0$ $f^{\prime}(\infty)=0, \quad \theta(\infty)=0, \quad \phi(\infty)=0$ as $\eta \rightarrow \infty$,

where $D a_{x}=\frac{k}{x^{2}}$ is the local Darcy number (Afify and Elgazery, 2013), $k_{1}=\frac{v}{k b}$ is the porous parameter, $H a=\sqrt{\frac{\sigma}{\rho b}} B_{0}$ is the Hartmann number, $E_{1}=\frac{E}{B_{0} b}$ is the local electric parameter, $F^{*}=\frac{C_{b}}{\sqrt{D a_{x}}}$ is the inertia-coefficient, $\lambda=\frac{G r_{x}}{\operatorname{Re}_{x}{ }^{2}}$ is the buoyancy or mixed convection parameter, $G r_{x}$ is the local Grashof uumber and $\operatorname{Re}_{\mathrm{x}}$ is the local Reynolds number. $\operatorname{Pr}=\frac{v}{\alpha} \quad$ is the Prandtl number, $E c=\frac{b^{2} l^{2}}{A_{0} C_{p}}$ is the Eckert number and $N r=\frac{16 \sigma^{*} T_{\infty}^{3}}{3 K \kappa}$ is the thermal radiation parameter, $D_{f}=\frac{D_{m} K_{T}\left(C_{w}-C_{\infty}\right)}{v C_{s} C_{p}\left(T_{w}-T_{\infty}\right)} \quad$ is the Dufour number. $S c=\frac{v}{D_{m}} \quad$ is the Schmidt number and $S r=\frac{D_{m} k_{T}\left(T_{w}-T_{\infty}\right)}{v T_{m}\left(C_{w}-C_{\infty}\right)}$ is the Soret number.

The physical quantities of interest are the skinfriction coefficient $\mathrm{C}_{\mathrm{f}}$, which is defined as

$$
C_{f}=\frac{\tau_{w}}{\rho U^{2} / 2}
$$

where wall sharing stress $\tau_{w}$ is given by $\tau_{w}=\mu\left(\frac{\partial u}{\partial y}\right)_{y=0}$.

Using the non-dimensional variables (6), we get from Eqs. (13) and (14) as

$\frac{1}{2} C_{f} \operatorname{Re}_{x}{ }^{1 / 2}=f^{\prime \prime}(0) \cdot$

The local Nusselt number which are defined as

$N u_{x}=\frac{x q_{w}}{\kappa\left(T_{w}-T_{\infty}\right)}$

where $q_{w}$ is the heat transfer from the sheet is given by

$q_{w}=-\left[\left(\frac{16 \sigma^{*} T^{3}}{3 k^{*}}+\kappa\right) \frac{\partial T}{\partial y}\right]_{y=0}$.

Using the non-dimensional variables (6), we get from Eqs. (16) and (17) as

$\frac{N u_{x}}{\operatorname{Re}_{x}^{1 / 2}}=-\left(1+N r \theta_{w}^{3}\right) \theta^{\prime}(0)$.

where $\theta_{w}$ is the temperature excess ratio parameter.

The physical quantity of interest is the local Sherwood number which are defined as

$S h_{x}=\frac{x q_{m}}{D_{m}\left(C_{w}-C_{\infty}\right)}$

where $q_{m}$ is the mass transfer which is defined by

$q_{m}=-D_{m}\left(\frac{\partial C}{\partial y}\right)_{y=0}$.

Using the non-dimensional variables (6), we get from Eqs. (19) and (20) as

$S h_{x} / \operatorname{Re}_{x}{ }^{1 / 2}=-\phi^{\prime}(0)$

where $\operatorname{Re}_{x}=\frac{x U_{w}(x)}{v}$ is the local Reynolds number

\section{NUMERICAL SOLUTIONS}

The coupled ordinary differential Eqs. (9) to (11) are of third-order in $\mathrm{f}$ and second-order in $\theta$ and $\phi$, which are first reduced to a system of seven simultaneous equations of first-order having seven unknowns. We adopt numerical method to solve the system of equations using fifth-order Runge-KuttaFehlberg integration scheme with shooting technique. The value of $\eta_{\infty}$ was selected to vary from 3 to 25 depending upon the physical parameters such as magnetic parameter, Prandtl number, thermal radiation parameter and Schmidt number so that no numerical oscillations would occur. The coupled nonlinear boundary value problem has been reduced to a system of seven simultaneous equations of first-order for seven unknowns as follows: 
$f_{1}^{\prime}=f_{2}, \quad f_{2}^{\prime}=f_{3}$,

$f_{3}^{\prime}=f_{2}^{2}-f_{1} f_{3}-H a^{2}\left(E_{1}-f_{2}\right)+k_{1} f_{2}$

$+F^{*} f_{2}^{2}-\lambda f_{4}-\delta f_{6}$,

$f_{4}^{\prime}=f_{5}$,

$f_{5}^{\prime}=\operatorname{Pr} /\left(1+N r-\operatorname{Pr} S c S r D_{f}\right)\left[\left(2 f_{2} f_{4}\right.\right.$

$\left.-f_{1} f_{5}\right)-E c f_{3}^{2}-E c H a^{2}\left(f_{2}-E_{1}\right)^{2}$

$-S c D_{f}\left(2 f_{2} f_{6}-f_{1} f_{7}\right)$

$f_{6}^{\prime}=f_{7}, f_{7}^{\prime}=-S c\left(f_{1} f_{7}-2 f_{2} f_{6}\right)$

$-\operatorname{Sc} \operatorname{Sr} f_{5}$

where

$f_{1}=f, f_{2}=f^{\prime}, f_{3}=f^{\prime \prime}, f_{4}=\theta$,

$f_{5}=\theta^{\prime}, f_{6}=\phi, f_{7}=\phi^{\prime}$,

and a prime denotes differentiation with respect to $\eta$. The boundary conditions now become

$f_{1}=0, f_{2}=1, f_{4}=1, f_{6}=1$, at $\eta=0$

$f_{2} \rightarrow \infty, f_{4} \rightarrow 0, f_{6} \rightarrow 0$, as $\eta \rightarrow \infty$.

Since $f_{3}(0), f_{5}(0)$ and $f_{7}(0)$ are not prescribed so we have to start with the initial guess value of $f_{3}(0)=s_{10}, f_{5}(0)=s_{20}$ and $f_{7}(0)=s_{30}$. Let $\gamma_{1}, \gamma_{2}$ and $\gamma_{3}$ be the correct values of $f_{3}(0), f_{5}(0)$ and $f_{7}(0)$, respectively. The resultant system of seven ordinary differential equations is integrated using fifth-order RungeKutta-Fehlberg method with shooting technique and denote the values of $f_{3}, f_{5}$ and $f_{7}$ at $\eta=\eta_{\infty}$ by $f_{3}\left(s_{10}, s_{20}, s_{30}, \eta_{\infty}\right), \quad f_{5}\left(s_{10}, s_{20}, s_{30}, \eta_{\infty}\right)$ and $f_{7}\left(s_{10}, s_{20}, s_{30}, \eta_{\infty}\right)$, respectively. Since $f_{3}, f_{5}$ and $f_{7}$ at $\eta=\eta_{\infty}$ are clearly function of $\gamma_{1}, \gamma_{2}$ and $\gamma_{3}$, they are expanded in Taylor series around $\gamma_{1}-s_{10}, \gamma_{2}-s_{20}$ and $\gamma_{3}-s_{30}$ , respectively by retaining only the linear terms. The use of difference quotients is made for the derivatives appeared in these Taylor series expansions. Thus, after solving the system of Taylor series expansions for $\delta \gamma_{1}=\gamma_{1}-s_{10}, \delta \gamma_{2}=\gamma_{2}-s_{20}$ and $\delta \gamma_{3}=\gamma_{3}-s_{30}$, we obtain the new estimates $s_{11}=s_{10}+\delta s_{10}, s_{21}=s_{20}+\delta s_{20} \quad$ and $s_{31}=s_{30}+\delta s_{30}$. Next the entire process is repeated starting with $f_{1}(0), f_{2}(0), s_{11}, f_{4}(0), s_{21}, f_{5}(0)$ and $S_{31}$ as initial conditions. Iteration of the whole outlined process is repeated with the latest estimates of $\gamma_{1}, \gamma_{2}$ and $\gamma_{3}$ until prescribed boundary conditions are satisfied. Finally, $s_{1 n}=s_{1(n-1)}+\delta s_{1(n-1)}, s_{2 n}=s_{2(n-1)}+\delta s_{2(n-1)}$ and $s_{3 n}=s_{3(n-1)}+\delta s_{3(n-1)}$ for $\mathrm{n}=1,2,3, \ldots$ are obtained which seemed to be the most desired approximate initial values of $f_{3}(0), f_{5}(0)$ and $f_{7}(0)$. In this way all the six initial conditions are determined. Now it is possible to solve the resultant system of seven simultaneous equations by fifthorder Runge-Kutta-Fehlberg integration scheme so that velocity, temperature and concentration fields for a particular set of physical parameters can easily be obtained. The results are provided in the form of table and graphs.

\section{RESULTS AND DISCUSSION}

Numerical solutions of the governing Eqs. (9) to (11) subject to boundary conditions (12) are obtained using Runge-Kutta-Fehlberg with shooting technique. The results are presented graphically in Figs. 2 to 21 and the conclusions are drawn for velocity, temperature and concentration field for various physical quantities of interest that have significant effects. Comparison of the present results of $-\theta^{\prime}(0)$ with those of Ishak et al. (2008) and Chen (1998) (see Table 1) in the absence of buoyancy force and magnetic field show a very good agreement.

Figures 2 to 4 depict the velocity, temperature and concentration profiles for different values of Dufour and Soret numbers. It is observed that the velocity and temperature profiles decrease by decreasing the Dufour number $D_{f}$ (or increasing the Soret number

$S_{r}$ ), whereas reverse effects are seen on the concentration profiles. The effects of Soret and Dufour numbers are significant near the stretching sheet for all velocity, temperature and concentration profiles.

Figures 5 to 8 represent the variations of velocity, temperature and concentration distributions in the boundary layer for various values of mixed convection parameter or thermal buoyancy parameter $\lambda$. Here, the positive values of thermal buoyancy parameter $\lambda$ correspond to cooling of the plate. Also, as the buoyancy parameter $\lambda$ increases, the peak of the velocity distribution increases rapidly near the vertical plate which decays smoothly to the free stream velocity. Reverse trend is seen on the temperature and concentration distributions, i.e., the effect of increasing the thermal buoyancy parameter $\lambda$ decreases the temperature and concentration profiles in the boundary layer due to decrease in the thickness of the thermal and solutal boundary-layers.

The solutal buoyancy parameter $\delta$ defines the ratio of the species buoyancy force to the viscous hydrodynamic force. As expected, the fluid velocity increases, and the peak value is more distinctive due to increase in the species of the solutal buoyancy parameter $\delta$ as seen from Fig. 8. Further, it is observed that the velocity distribution attains a distinctive maximum value in the vicinity of the plate which ultimately decreases to the free stream value. From Figs. 9 and 10, it is observed that the temperature and concentration profiles decrease 
with increasing the solutal buoyancy parameter $\delta$.

Figures 11 and 12 depict the graph of nondimensional temperature and concentration profiles for different values of Eckert number. By analyzing the graph, it is revealed that the effect of Eckert number $\mathrm{Ec}$ is to increase the temperature distribution in the flow region. This is because heat energy is stored in the liquid due to the frictional heating, which causes the temperature to increase in the thermal boundary layer. Thus the effect of increasing $\mathrm{Ec}$ is to enhance the temperature at any point whereas reverse trend is seen on concentration profiles.

The influence of thermal radiation parameter $\mathrm{Nr}$ on the tangential velocity is shown in Fig. 13. It is seen from this figure that increase in the thermal radiation parameter produces an increase in the tangential velocity of the flow. This is because large values of $\mathrm{Nr}$ correspond to increased velocity profiles. The effects of radiation parameter $\mathrm{Nr}$ on temperature and concentration profiles are shown in Figs. 14 and 15 . The effect of $\mathrm{Nr}$ is prominently seen throughout the boundary layer. From Fig. 14 it is observed that the effect of $\mathrm{Nr}$ is to increase the temperature distribution in the thermal boundary layer. This is due to the fact that increase in the value of $\mathrm{Nr}$ implies increasing of radiation in the thermal boundary layer and hence increases the value of temperature profile in the thermal boundary layer. Figure 15 depicts the effect of the thermal radiation parameter $\mathrm{Nr}$ on the concentration profiles. It is observed that an increase in the thermal radiation parameter $\mathrm{Nr}$ leads to decrease in the concentration profile.

The graph of concentration profiles $\phi(\eta)$ for different values of Schmidt number Sc is plotted in Fig. 16. The analysis of the graph reveals that the effect of increasing the value of Schmidt number Sc is to decrease concentration distribution in the flow region. Physically, the increase of $\mathrm{Sc}$ means decrease of molecular diffusivity, which results in decrease in the thickness of the concentration boundary layer. Hence, the concentration of species is more for small values of $\mathrm{Sc}$ and lower for higher values of Schmidt number in the solutal boundary layer.

The effect of magnetic field on the local skin friction coefficient, in terms of $f^{\prime \prime}(0)$, is displayed in Fig. 17. It is observed that the local local skin friction coefficient decreases with increasing the Hartman number $\mathrm{Ha}$, whereas no change is observed by increasing the Schmidt number Sc. It is observed from Fig. 18 that the local Nusselt number, in terms of $-\theta^{\prime}(0)$ increases with increase in the magnetic field strength and Schmidt number Sc.

Figures 19 to 21 depict the effect of local inertia coefficient $F^{*}$ and Schmidt number Sc on the skinfriction coefficient, local Nusselt number and local Sherwood number, respectively. From these figures, it is analyzed that the behavior of $f^{\prime \prime}(0),-\theta^{\prime}(0)$ and $-\phi^{\prime}(0)$ changes with increase in the values of $F^{*}$ and Sc. Thus we observe that the local skin friction coefficient decreases with increasing the value of $F^{*}$. Further, the effect of increasing the value of Schmidt number Sc on the local Nusselt number is to increase its value and similar effect is seen on the Sherwood number which increases with increase in the value of $F^{*}$. The effect of $\mathrm{Sc}$ is prominently seen on Nusselt number and Sherwood number for all the values of $F^{*}$, i.e., local Nusselt number increases with increase in the value of Schmidt number and reverse effect is seen on the local Sherwood number.

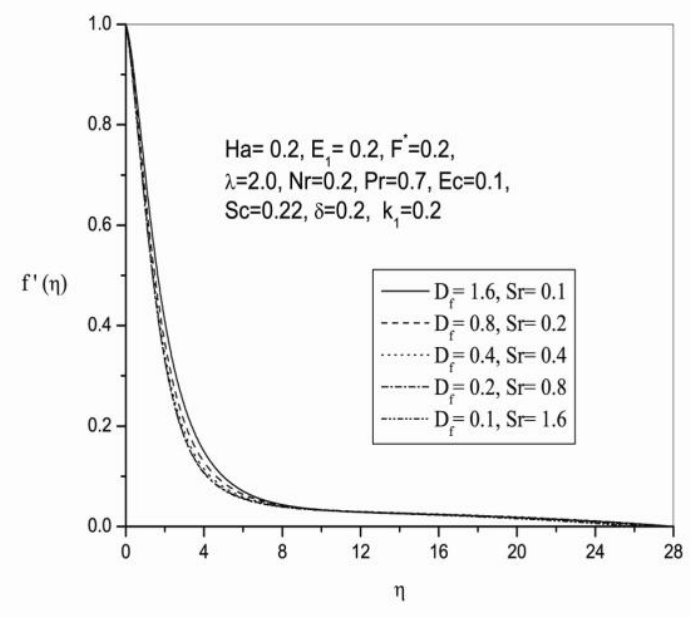

Fig. 2. Influence of Soret and Dufour parameters on the dimensionless velocity profiles.

Table 1 Comparison of the local Nusselt number $-\theta^{\prime}(0)$ with Ishak et al. (2008) and Chen (1998) for various values of $\mathrm{Pr}$ and $H a=D_{f}=S r=F^{*}=N r=E c=$ $k_{1}=E_{1}=\lambda=\delta=S c=0$.

\begin{tabular}{|c|l|l|l|}
\hline Pr & $\begin{array}{l}\text { Ishak } \\
\text { et al. (2008) }\end{array}$ & $\begin{array}{l}\text { Chen } \\
(1998)\end{array}$ & $\begin{array}{l}\text { Present } \\
\text { Results }\end{array}$ \\
\hline 1.0 & 1.3333 & 1.33334 & 1.333333 \\
\hline 3.0 & 2.5097 & 2.50997 & 2.509715 \\
\hline 10.0 & 4.7969 & 4.79689 & 4.796871 \\
& & & \\
\hline
\end{tabular}




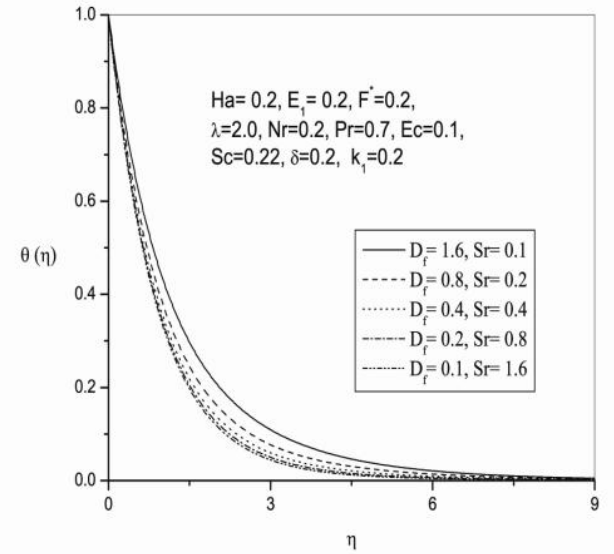

Fig. 3. Influence of Soret and Dufour parameters on the dimensionless temperature profiles.

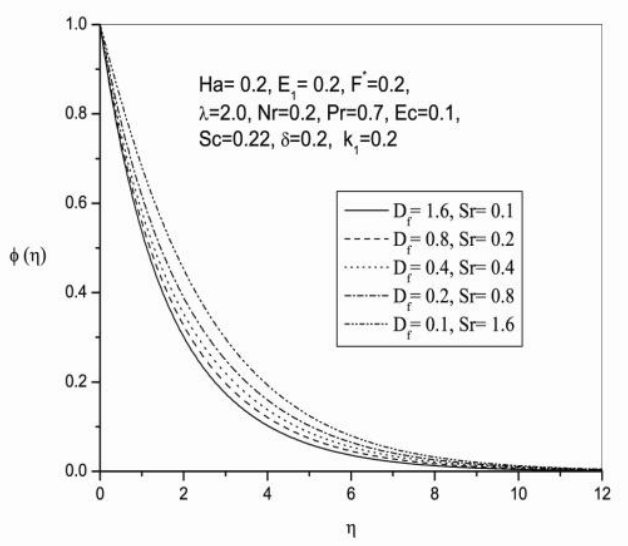

Fig. 4. Influence of Soret and Dufour parameters on the dimensionless concentration profiles.

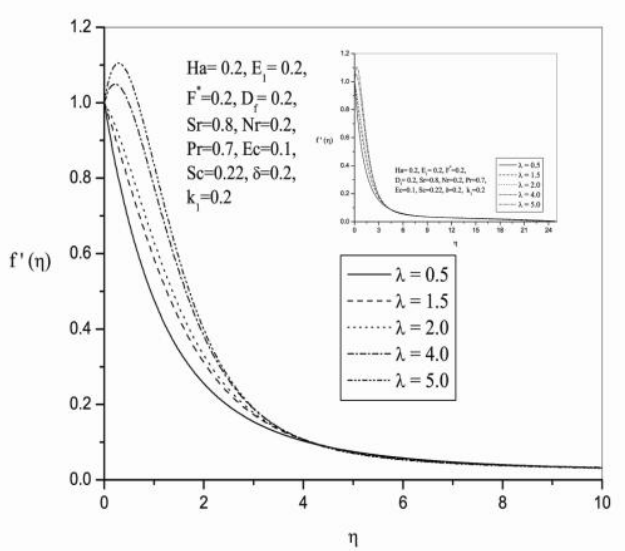

Fig. 5. Influence of the buoyancy parameters $\lambda$ on the dimensionless velocity profile.

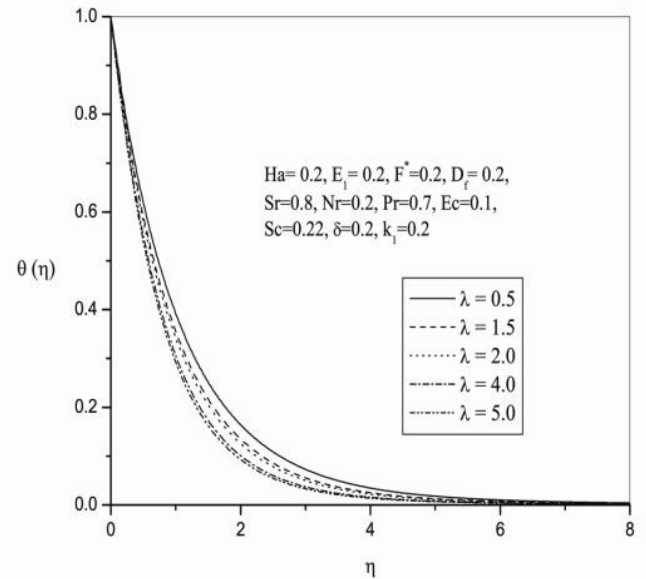

Fig. 6. Influence of the buoyancy parameters $\lambda$ on the dimensionless temperature profile.

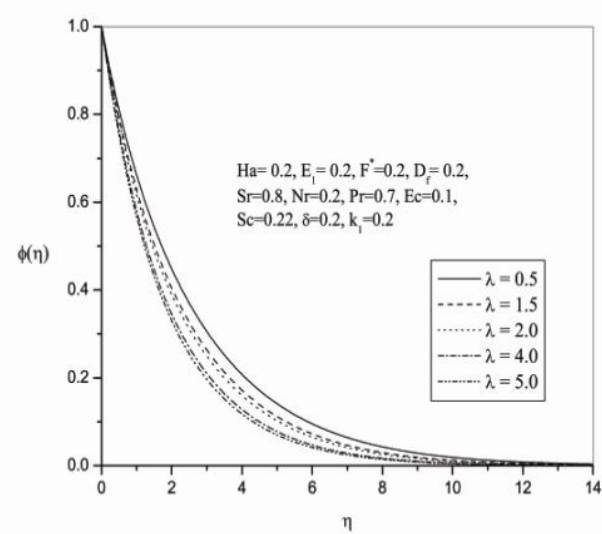

Fig. 7. Influence of the buoyancy parameters $\lambda$ on the dimensionless concentration profile.

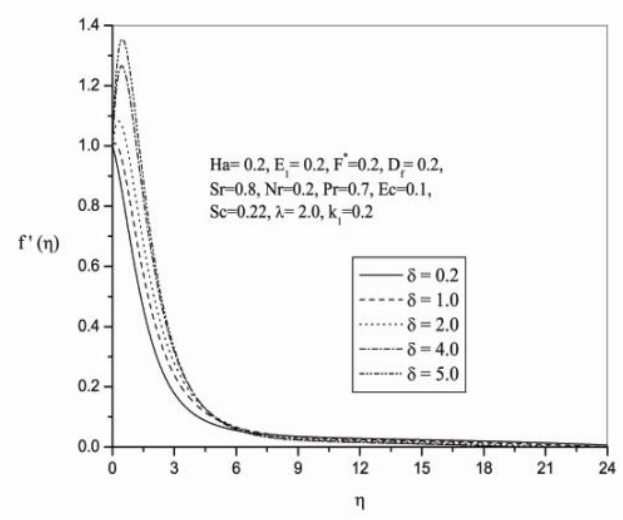

Fig.8. Influence of the solutal buoyancy parameters $\delta$ on the dimensionless velocity profile. 


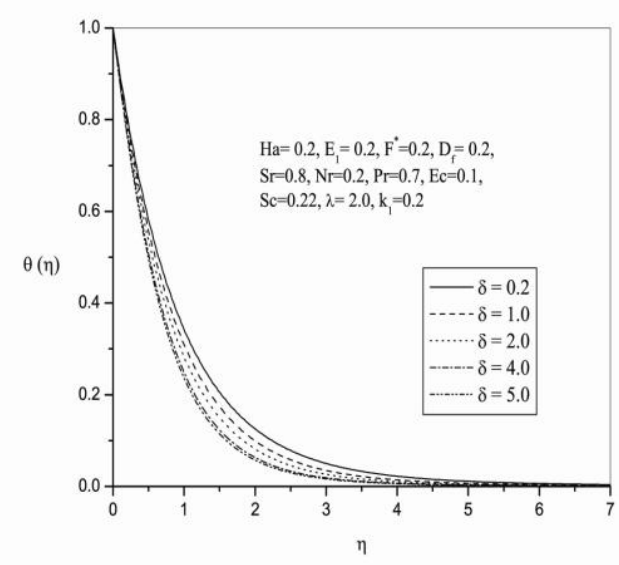

Fig. 9. Influence of the solutal buoyancy parameters $\delta$ on the dimensionless temperature profile.

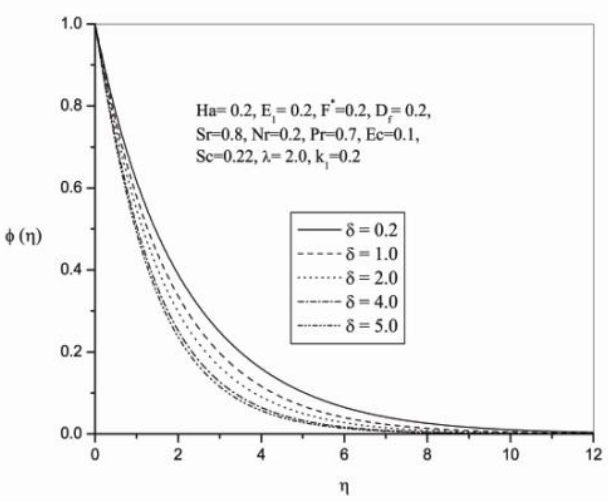

Fig. 10. Influence of the solutal buoyancy parameters $\delta$ on the dimensionless concentration profile.

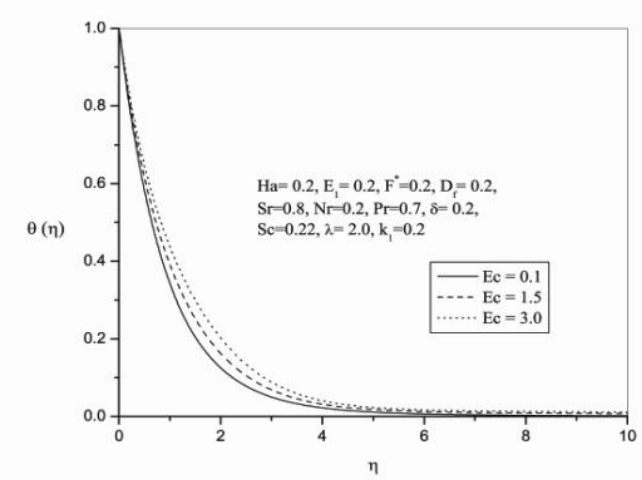

Fig. 11. Influence of the Eckert number $E c$ on the dimensionless temperature profile.

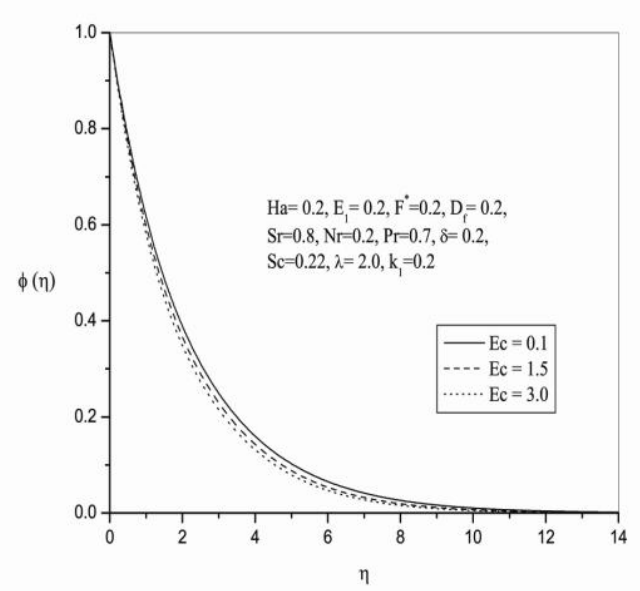

Fig. 12. Influence of the Eckert number $E c$ on the dimensionless concentration profile.

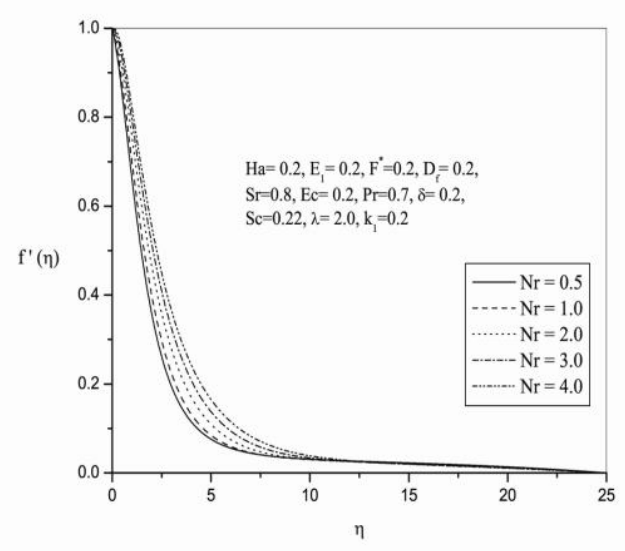

Fig. 13. Influence of the thermal radiation parameter $\mathrm{Nr}$ on the dimensionless velocity profile.

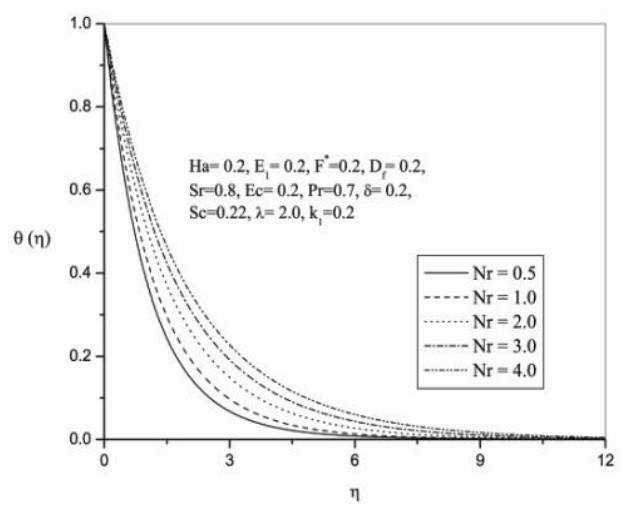

Fig. 14. Influence of the thermal radiation parameter $\mathrm{Nr}$ on the dimensionless temperature profile. 


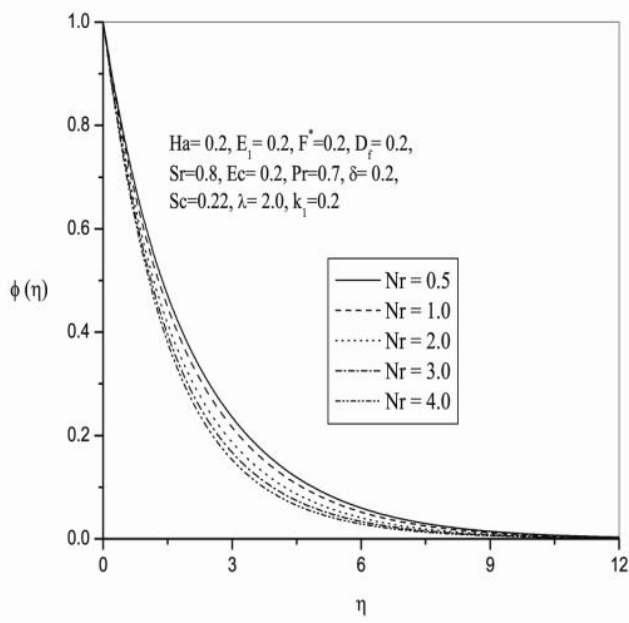

Fig. 15. Influence of the thermal radiation parameter $\mathrm{Nr}$ on the dimensionless concentration profile.

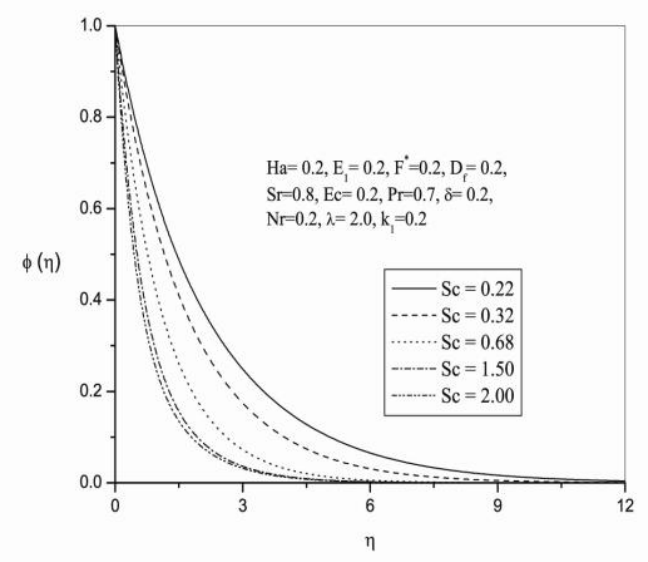

Fig. 16. Influence of the Schmidt number $S c$ on the dimensionless concentration profile.

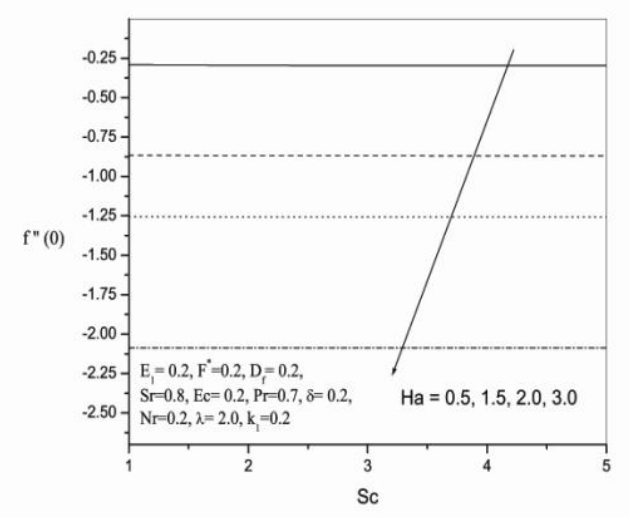

Fig. 17. Influence of $S c$ on skin friction coefficient for various values of $\mathrm{Ha}$.

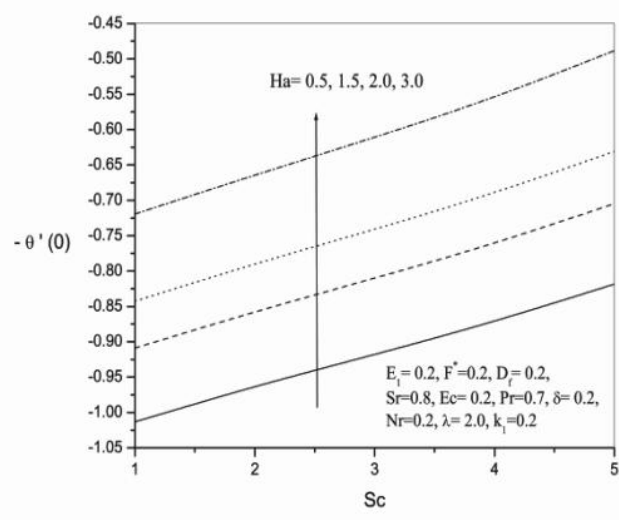

Fig. 18. Influence of $S c$ on nusselt number for various values of $\mathrm{Ha}$.

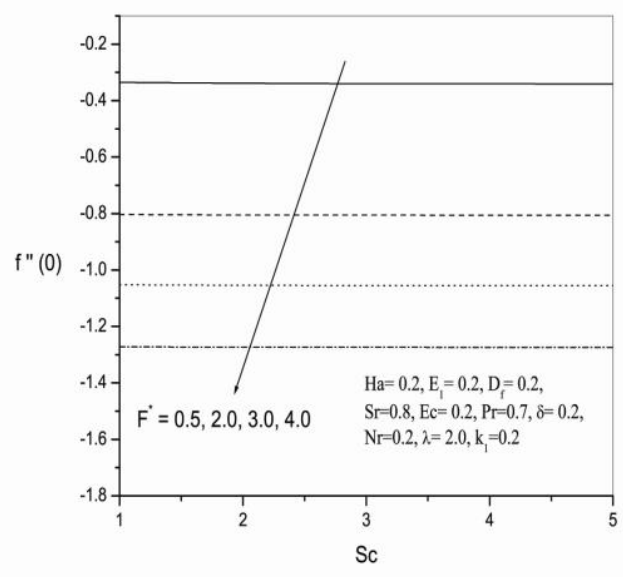

Fig. 19. Influence of $S c$ on skin friction coefficient for various values of $\mathbf{F}^{*}$.

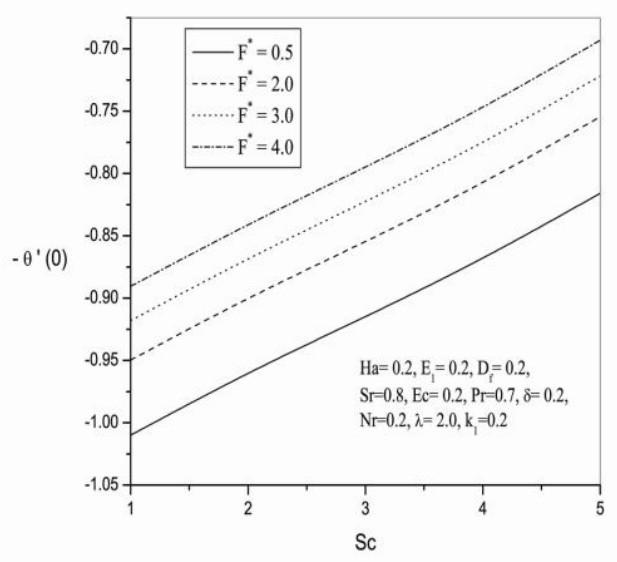

Fig. 20. Influence of $S \boldsymbol{c}$ on Nusselt number for various values of $F^{*}$. 


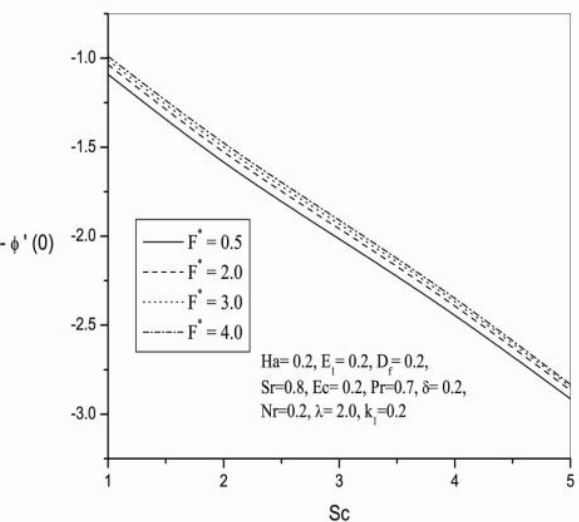

Fig. 21. Influence of $S c$ on Sherwood number for various values of $F^{*}$.

\section{CONCLUSION}

An analysis has been carried out to study non-Darcy boundary layer flow, heat and mass transfer characteristics in an incompressible electrically conducting fluid over a linear stretching sheet with transverse magnetic field, radiation, viscous dissipation, Ohmic heating in the presence of Soret and Dufour effects. The effects of various physical parameters like Schmidt number, Eckert number, Hartmann number, Soret and Dufour parameters on velocity, temperature, and concentration profiles are obtained. The following conclusions are drawn from the present study:

(i) Increasing the porous parameter decreases the velocity distribution in the boundary layer.

(ii) Temperature profiles are strongly influenced by the thermal radiation parameter i.e., temperature increases with increase in the thermal radiation parameter.

(iii) Increase in solutal buoyancy parameter $\delta$ increases the velocity distribution with formation of peak for higher values of solutal buoyancy parameter in the solutal boundary layer.

(iv) Increasing thermal buoyancy parameter is to increase velocity profiles and decrease temperature and concentration profiles.

(v) Skin-friction coefficient decreases with increase in the value of Hartmann number $\mathrm{Ha}$ whereas reverse effect is seen the local on Nusselt number.

(vi) Inclusion of local inertia coefficient effect $\mathrm{F}^{*}$ is to decrease local skin-friction coefficient and increases the local Sherwood number and the local Nusselt number.

\section{REFERENCES}

Afify AA and Elgazery NS (2013) Effect of double dispersion on non-Darcy mixed convective flow over vertical surface embedded in porous medium. Applied Mathematics and Mechanics- Engl. Ed.

\section{DOI $10.1007 / s 10483-013-1741-8$}

Alam S, Rahman MM, Maleque A and Ferdows M (2006) Dufour and Soret effects on steady MHD combined free-forced convective and mass transfer flow past a semi-infinite vertical plate, Thammasat Int J Sci Tech, 11(2), 112.

Ali ME (2007) The effect of lateral mass flux on the natural convection boundary layers induced by a heated vertical plate embedded in a saturated porous medium with internal heat generation, Int J Thermal Sci (46) 157 163.

Anjalidevi SP and Kayalvizhi M (2013) Nonlinear hydromagnetic flow with radiation and heat source over a stretching surface with prescribed heat and mass flux embedded in a porous medium. Journal of Applied Fluid Mechanics 6(2) 157-165.

Bakier AY and Gorla RSR (1996) Thermal radiation effect on mixed convection from horizontal surfaces in saturated porous media, Transport in Porous Media (23) 357-363.

Bejan A and Poulikakos D (1984) The non-Darcy regime for vertical boundary layer natural convection in a porous medium, Int $J$ Heat Mass Transfer ,(27), 717-722.

Chen CH (1998) Laminar mixed convection adjacent to vertical continuously stretching sheets, Heat Mass Transfer (33) 471-476.

Crane L J (1970), Flow past a stretching plate, $Z$ Angew Math Phys. (21) 645-647.

Gangadha K (2013) Soret and Dufour effects on hydro magnetic heat and mass transfer over a vertical plate with a convective surface boundary condition and chemical reaction, Journal of Applied Fluid Mechanics, 6( 1), 95-105.

Ishak A, Nazar R and Pop I (2008) Hydromagnetic flow and heat transfer adjacent to a stretching vertical sheet, Heat Mass Transfer, (44), 921927.

Minto BJ, Ingham DB and Pop I (1998) Free convection driven by an exothermic reaction on a vertical surface embedded in porous media, Int J Heat Mass Transfer (41), 1124. 
D. Pal1 and H. Mondal/ JAFM, Vol. 7, No. 3, pp. 513-523, 2014.

Mostafa AAM and Shimaa EW (2012) MHD flow and heat transfer of a micropolar fluid over a stretching surface with heat generation/absorption and slip velocity, $J$ Egyptian Mathematical Soc (20), 20-27.

$\mathrm{Na}$ TY (1979) Computational method in engineering boundary value problems, New York; Academic Press.

Nield DA and Bejan A (2006) Convection in porous media. 3rd ed. New York: Springer.

Pal D and Malashetty MS (2008) Radiation effects on stagnation-point flow over a stretching sheet with internal heat generation or absorption, Int J Appl Mech Eng (13), 427-39.

Pal D (2009) Heat and mass transfer in stagnationpoint flow towards a stretching surface in the presence of buoyancy force and thermal radiation, Meccanica (44), 145-158.

Pal D and Mondal H (2011) The influence of thermal radiation on hydromagnetic DarcyForchheimer mixed convection flow past a stretching sheet embedded in a porous medium. Meccanica, (46), 739-753.

Pal D and Chatterjee S (2011) Mixed convection magnetohydrodynamic heat and mass transfer past a stretching surface in a micropolar fluidsaturated porous medium under the influence of Ohmic heating, Soret and Dufour effects, Commun Nonlinear Sci Numer Simulat, (16)
1329-1346.

Postelnicu A (2004) Influence of a magnetic field on heat and mass transfer by natural convection from vertical surfaces in porous media considering Soret and Dufour effects, Int J Heat Mass Trans, (47), 1467--1472.

Reddy PS, Rao VP (2012) Thermo-Diffusion and Diffusion -Thermo Effects on Convective Heat and Mass Transfer through a Porous Medium in a Circular Cylindrical Annulus with Quadratic Density Temperature Variation - Finite Element Study, Journal of Applied Fluid Mechanics, 5( 4), 139-144.

Rudraiah N (1984) Non-linear convection in a porous medium with convective acceleration and viscous force, Arab J Sci Eng, (9), 153--67.

Vafai K and Kim SJ (1989) Forced convection in a channel filled with porous medium: an exact solution, ASME J Heat Transfer, (111), 1103-6.

Vafai K and Tien CL (1981) Boundary and inertia effects on flow and heat transfer in porous media, Int J Heat Mass Transfer, (24), 195-203.

Vajravelu K and Rollins D (1992) Heat transfer in electrically conducting fluid over a stretching sheet. Int J Non-linear Mech, 27(2), 265277. 